\title{
Trend of Mortality and Years of Life Lost Due to Chronic Obstructive Pulmonary Disease in China and Its Provinces, 2005-2020
}

\author{
Wei Liu (D* \\ Wei Wang* \\ Jiangmei Liu \\ Yunning Liu \\ Shidi Meng (D) \\ Feixue Wang (D) \\ Zheng Long (D) \\ Jinlei Qi \\ Jinling You \\ Lin Lin \\ Lijun Wang \\ Maigeng Zhou \\ Peng Yin
}

National Center for Chronic and Noncommunicable Disease Control and Prevention, Chinese Center for Disease Control and Prevention, Beijing, People's Republic of China

*These authors contributed equally to this work
Correspondence: Peng Yin

National Center for Chronic and

Noncommunicable Disease Control and

Prevention, Chinese Center for Disease

Control and Prevention, No. 27 Nanwei

Road, Beijing, 100050, People's Republic of China

Tel +86 010-630I5058

Email yinpeng@ncncd.chinacdc.cn
Background: To examine trends in chronic obstructive pulmonary disease (COPD) mortality and years of life lost (YLL) due to COPD for all provinces in China during 2005-2020. Methods: Data for COPD mortality were derived from China National Mortality Surveillance System (NMSS). We analyzed the numbers and age-standardized rates of death and YLL due to COPD in China, during 2005-2020. We carried out decomposition analysis to analyze the drivers of change in COPD deaths during the study period.

Results: The age-standardized mortality rate of COPD in China decreased significantly from $99.5 / 100,000$ in 2005 to $50.5 / 100,000$ in 2020. Similar trend was seen in the age-standardized YLL rate. The mortality rate increased with age. During 2005-2020, the age-standardized mortality rate decreased in all provinces (except for Tibet) with the largest decline in Jilin (-77.8\%), Henan (-68.4\%) and Fujian (-67.1\%). The decreased number of deaths was decomposed as population growth $(8.5 \%)$ and population ageing $(69.7 \%)$ with offset by decline of age-specific mortality $(-87.5 \%)$.

Conclusion: COPD remains an important public health problem in China, though significant reductions of COPD mortality and YLL rate were observed. Vigorous prevention and control strategies should be enhanced to improve the quality of life of COPD patients and reduce the premature death caused by COPD in Chinese population.

Keywords: COPD, mortality, YLL

\section{Introduction}

Chronic obstructive pulmonary disease (COPD) is a leading cause of mortality worldwide and in China. ${ }^{1}$ Previous studies have shown significant decline of COPD mortality through time for both sexes at national and provincial level in China before year 2013. ${ }^{2,3}$ Despite the declining trend, COPD mortality remains high in China, particularly in some western provinces. It is important to appreciate that the decline in COPD mortality did not occur equally across all regions, as shown in a study using data from disease surveillance point system in China, which also indicated urban/rural differences in COPD mortality narrowed over time, but the magnitude of the east-west inequality persisted without change during 2006-2012. ${ }^{3}$

China is experiencing the most rapid development ever seen in history and it is therefore important to update the status of COPD mortality at provincial level from a policy perspective as the policy makers are required to allocate limited health resources efficiently based on the most recent evidence and develop or adjust tailored intervention strategies with thorough understanding of the current 
epidemiological data. In addition, the years of life lost (YLL) has been more and more commonly used to characterize premature death and disease burden compared with mortality, because it could take the death count and life expectancy at death into consideration. YLL estimates the years of potential life lost due to premature deaths and can be used in public health planning to compare the relative importance of different causes of premature deaths within a given population, to set priorities for prevention, and to compare the premature mortality experience between populations. However, no studies have examined the YLLs due to COPD at provincial level in China yet. The aims of this study were to examine trends in COPD mortality and YLL due to COPD for all provinces in China during 2005-2020 using data from China National Mortality Surveillance System (NMSS).

\section{Methods}

\section{Data Sources}

Data for COPD mortality were derived from the National Mortality Surveillance System (NMSS) with national and provincial representativeness. The system has been described in details elsewhere. ${ }^{4}$ Briefly, the system collects deaths occurred in hospital and outside-of-hospital through an online reporting platform, covering over 300 million population from 605 districts in urban or counties in rural in 31 provincial level administrative regions that account for $24 \%$ of the total population in China. Data for underreporting in this system were obtained from retrospective under-reporting field surveys for NMSS conducted in 2009, 2012, 2015 and 2018, aiming to derive under-reporting rate for period of 2006-2008, 2009-2011, 2012-2014 and 2015-2017, respectively. ${ }^{5}$ Given the relatively higher under-reporting rate among children in NMSS, we used Under-5 Mortality Rate (U5MR) at county level from the Global Burden of Disease Study as a separate data source. ${ }^{6}$ Data for surveillance population and socio-economic covariates were all from China National Bureau of Statistics. A flow chart of the estimation for COPD mortality is shown in Figure 1.

\section{All-Cause Mortality Estimation}

Annual under-reporting rate for each age-sex stratum among all surveillance sites during 2006-2017 was used to adjust the mortality rate. We used splines regression to predict under-reporting rate in each stratum in 2005 and 2018. To produce a complete set of age-specific mortality rate (an abridged life table) for each surveillance site, we used a new relational model life table system with flexible standard (MLTFS) based on two parameters of $5 \mathrm{q} 0$ and $45 q 15$ to generate a full set of age-sex-specific mortality rate for 31 provinces during 2005-2018. ${ }^{7}$ We fitted generalized linear model over time to predict all-cause mortality in 2019 and 2020.

\section{COPD Mortality Estimation}

Garbage codes, deaths with non-specific codes, deaths assigned to International Classification of Diseases (ICD) codes that could not be underlying causes of death, or deaths assigned to intermediate but not underlying causes of death were redistributed by age, sex, location, and year to the most likely causes of death based on the methods developed by Naghavi and colleagues. ${ }^{8}$ Proportion of COPD (defined as ICD-10 code J40-44) from NMSS by province-age-sex was calculated after garbage code redistribution. Mortality rate of COPD by each year, location, sex and age group was then calculated by multiplying allcause mortality rate by proportion of COPD in all deaths. Assuming a steady change for COPD mortality, we fitted generalized linear model to predict COPD mortality rate in 2019 and 2020 based on results for COPD mortality in each location-sex-age group stratum from 2005 to 2018.

\section{Years of Life Lost Estimation}

Years of life lost (YLLs) is a metric of premature death calculated as the sum of each death multiplied by the standard life expectancy at each age. We used a theoretical minimum risk reference life table, which was taken from the lowest observed risk of death for each 5-year age group in all population greater than 5 million as standard life expectancy in YLL estimation for COPD during 2005-2020. ${ }^{9}$

\section{Statistical Analysis}

We analyzed the number of death and YLL due to COPD and their age-standardized rates in all 31 provinces in China, during 2005-2020. The age-standardized mortality and YLL rates (per 100,000) were calculated through direct age standardization with Chinese sixth census in 2010 as the standard population. ${ }^{10}$ We used Joinpoint Regression Program ${ }^{11}$ (Version 4.8.0.1 - April 2020) to calculate the average annual percent change (AAPC) in age-standardized mortality and YLL rate during 2005-2020. To analyze the drivers of change in the numbers of COPD deaths by geography, we decomposed 


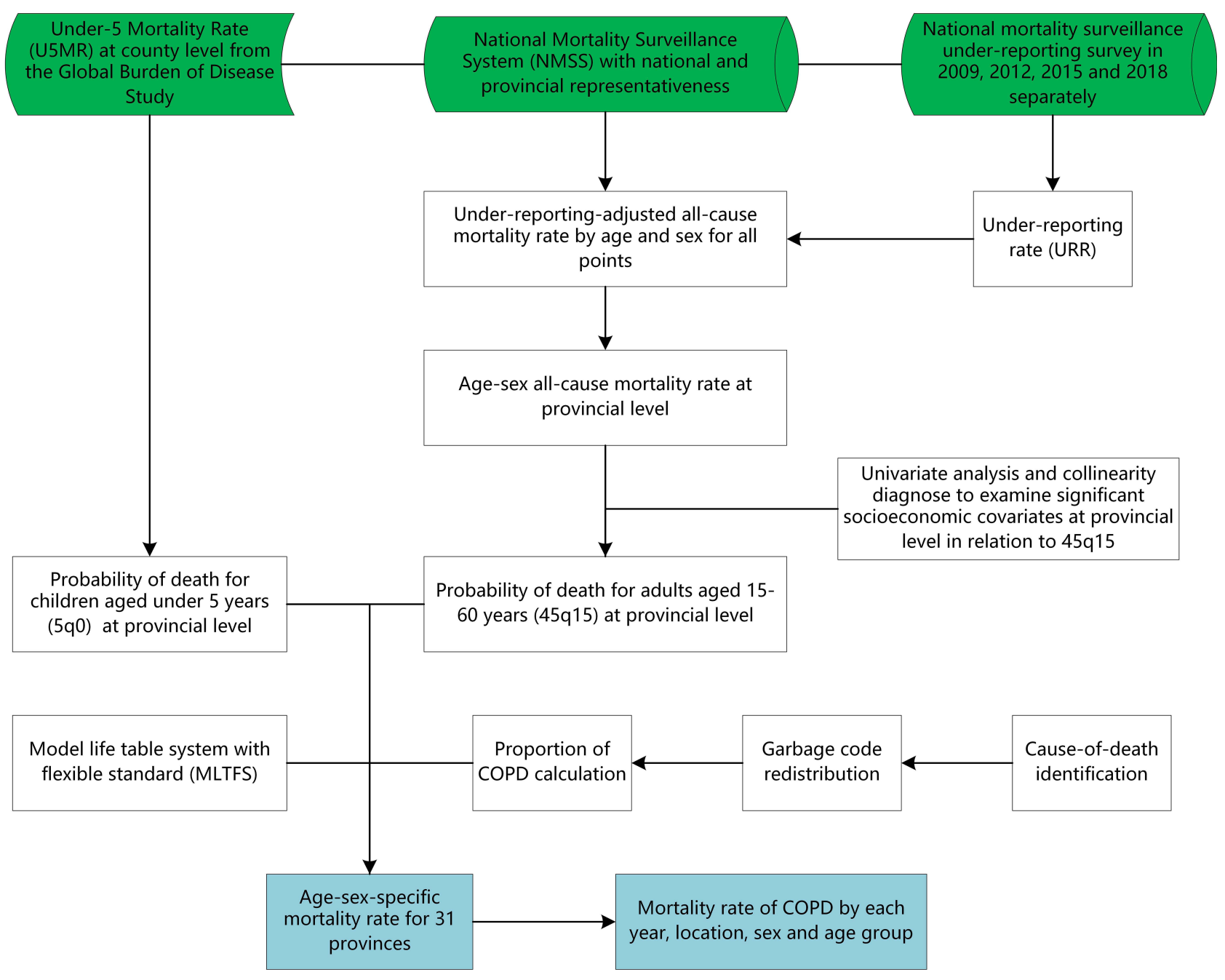

Figure I Flow chart of the estimation for all-cause mortality and COPD mortality.

Abbreviations: COPD, chronic obstructive pulmonary disease; U5MR, under-5 mortality rate; NMSS, National Mortality Surveillance System; URR, under-reporting rate; $45 q 15$, probability of death for adults aged I5-60 years; 5q0, probability of death for children aged under 5 years; MLTFS, model life table system with flexible standard.

change from 2005 to 2020 into three explanatory components: change due to growth of the total population; change in the population structure by age; and change in age-specific rates. $^{12}$ The decomposition analysis uses methods developed in demographic research by Das Gupta. ${ }^{13}$ We used counterfactual scenarios to calculate two different sets of numbers for death. In the first scenario, for population growth, it is assumed that the total population of the whole country and provinces in 2018 is the actual level, and the population structure and rates of death were the same in 2020 as in 2005 , the expected number of deaths in 2020 is calculated from the total population in 2020 and the population structure and agespecific mortality in 2005. In the second scenario, for population growth and ageing, it is assumed that the total population of the whole country and provinces and the population structure in 2020 is the actual level, but the age-specific rates of death were held constant to 2005. The contribution of population growth to the change of the total deaths is the difference between the estimated number of deaths in 2018 in the first scenario and the actual number of deaths in 2005. The contribution of population aging to the change of the total deaths is the difference between the second scenario and the first scenario. The contribution of age-specific mortality is the difference between the actual number of deaths in 2020 and the second scenario. Our analyses were carried out with SAS version 9.4 (SAS Institute Inc., Cary, NC, USA).

\section{Results}

As shown in Table 1, COPD accounted for 954,881 deaths (556,867 males, 398,014 females) and 14,764,545 YLL 
Table I Estimated Death and YLLs of COPD in China

\begin{tabular}{|c|c|c|c|c|}
\hline & Number of Death & $\begin{array}{c}\text { Age-Standardized Death } \\
\text { Rate }(1 / 100,000)\end{array}$ & Number of YLL & $\begin{array}{c}\text { Age-Standardized YLL Rate } \\
((1 / 100,000)\end{array}$ \\
\hline \multicolumn{5}{|l|}{ Male } \\
\hline 2005 & 545,121 & 115.9 & $9,787,321$ & 1957.2 \\
\hline 2010 & 555,539 & 97.9 & $9,582,667$ & 1629.9 \\
\hline 2015 & 543,010 & 80.7 & $8,986,272$ & 1317.0 \\
\hline 2020 & 556,867 & 66.8 & $9,029,966$ & 1068.7 \\
\hline AAPC (\%) & - & $-3.6(-3.8 \text { to }-3.4)^{\mathrm{a}}$ & - & $-4.0(-4.2 \text { to }-3.8)^{\mathrm{a}}$ \\
\hline \multicolumn{5}{|l|}{ Female } \\
\hline 2005 & 508,167 & 85.1 & $7,927,837$ & 1337.0 \\
\hline 2010 & 478,649 & 67.5 & $7,283,828$ & 1047.6 \\
\hline 2015 & 418,347 & 50.4 & $6,|87,49|$ & 767.2 \\
\hline 2020 & 398,014 & 37.0 & $5,734,579$ & 547.6 \\
\hline AAPC (\%) & - & $-5.5(-5.8 \text { to }-5.1)^{\mathrm{a}}$ & - & $-5.9(-6.2 \text { to }-5.5)^{\mathrm{a}}$ \\
\hline \multicolumn{5}{|l|}{ Total } \\
\hline 2005 & $\mathrm{I}, 053,288$ & 99.5 & $17,7 \mid 5,158$ & 1634.7 \\
\hline 2010 & $\mathrm{I}, 034,188$ & 81.7 & $16,866,495$ & 1326.3 \\
\hline 2015 & 961,357 & 64.6 & $15,173,763$ & 1029.4 \\
\hline 2020 & 954,881 & 50.5 & $14,764,545$ & 789.6 \\
\hline AAPC (\%) & - & $-4.5(-4.7 \text { to }-4.2)^{\mathrm{a}}$ & - & $-4.8(-5.1 \text { to }-4.5)^{\mathrm{a}}$ \\
\hline
\end{tabular}

Note: ${ }^{a} \mathrm{P}<0.01$.

Abbreviations: COPD, chronic obstructive pulmonary disease; AAPC, average annual percent change; YLL, years of life lost.

(9,029,966 males, 5,734,579 females) in China in 2020. The age-standardized death rate of COPD decreased from $99.5 / 100,000$ in 2005 to $50.5 / 100,000$ in 2020 , and the YLL rate decreased from $1634.7 / 100,000$ in 2005 to $789.6 / 100,000$ in 2020. The age-standardized death rate and YLL rate were consistently higher in males than in females, both with a declining trend during 2005-2020.

The age-standardized mortality rates of COPD in China decreased significantly in males (AAPC (average annual percent change) $=-3.6,95 \%$ CI: $-3.8 \sim-3.4$ ), females (AAPC $=-5.5,95 \%$ CI: -5.8 to -5.1 ) and both sexes $(\mathrm{AAPC}=-4.5,95 \% \mathrm{CI}:-4.7$ to -4.2$)$ from 2005 to 2020 (Figure 2A). Figure 2B shows the trend of agestandardized YLL rate of COPD which has the same trend as the age-standardized death. The age-standardized mortality rate of COPD for males, females and both sexes decreased by $42.4 \%, 56.5 \%$ and $49.2 \%$ respectively between 2005 and 2020 in China and the age-standardized YLL rate of COPD for males, females and both sexes decreased by $45.4 \%, 59.0 \%$ and $51.7 \%$ respectively.

The mortality rate of COPD increased with age and the age-specific mortality rate was decreasing across all age groups from 2005, 2010, 2015 to 2020 (Figure 3). The mortality rate of people aged above 80 years old was $3,620.4 / 100,000$ in 2005 and 2047.3/100,000 in 2020 .
As shown in Figure 4, Sichuan (123.7/100,000), Yunnan (116.3/100,000), and Qinghai $(95.7 / 100,000)$, all located in the western China, had the highest age-standardized death rates in 2020. Jilin $(9.7 / 100,000)$, Liaoning $(15.3 / 100,000)$, and Beijing $(18.6 / 100,000)$ had the lowest age-standardized death rates in 2020. Between 2005 and 2020 , the standardized mortality rate of almost all provinces except Tibet (33.4\%) decreased and Jilin (-77.8\%), Henan $(-68.4 \%)$ and Fujian (-67.1) showed the largest decline. The age-standardized YLL rate in different provinces was similar to that in age-standardized mortality rate.

Decomposition analyses for 2005 to 2020 are shown in Figure 5. The increase due to population change and population ageing was offset by decline of age-specific mortality rate at national level. From 2005 to 2020, the total number of deaths due to COPD in China decreased by $9.3 \%$, of which age-specific mortality accounted for $-87.5 \%$, population growth accounted for $8.5 \%$, and population aging accounted for $69.7 \%$. At the provincial level, the province with the greatest contribution to the decline in age-specific mortality is Jilin $(-158.2 \%)$, followed by Shanghai $(-140.7 \%)$ and Liaoning $(-129.0 \%)$, and the province with the lowest contribution is Tibet $(40.7 \%)$, followed by Hunan (- 39.7\%) and Hubei (- 45.4\%). The 
A

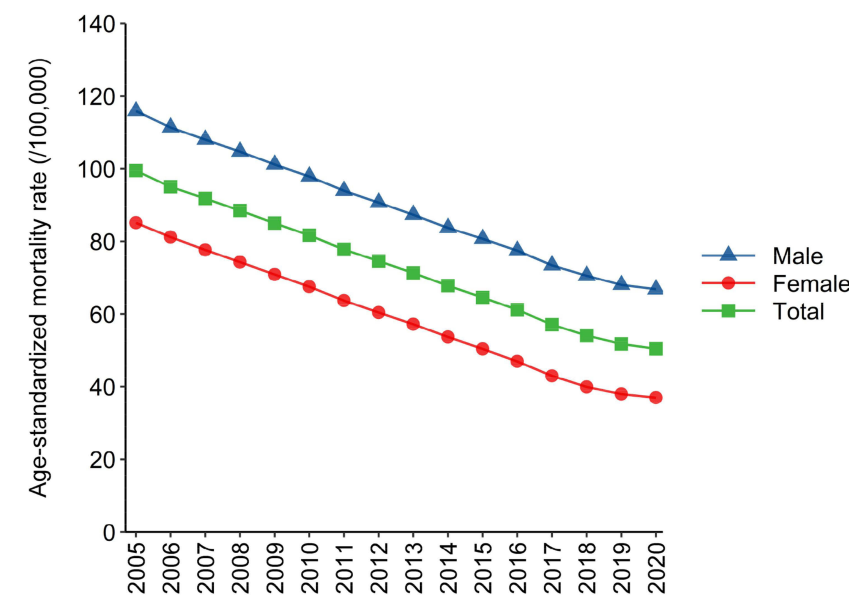

Year

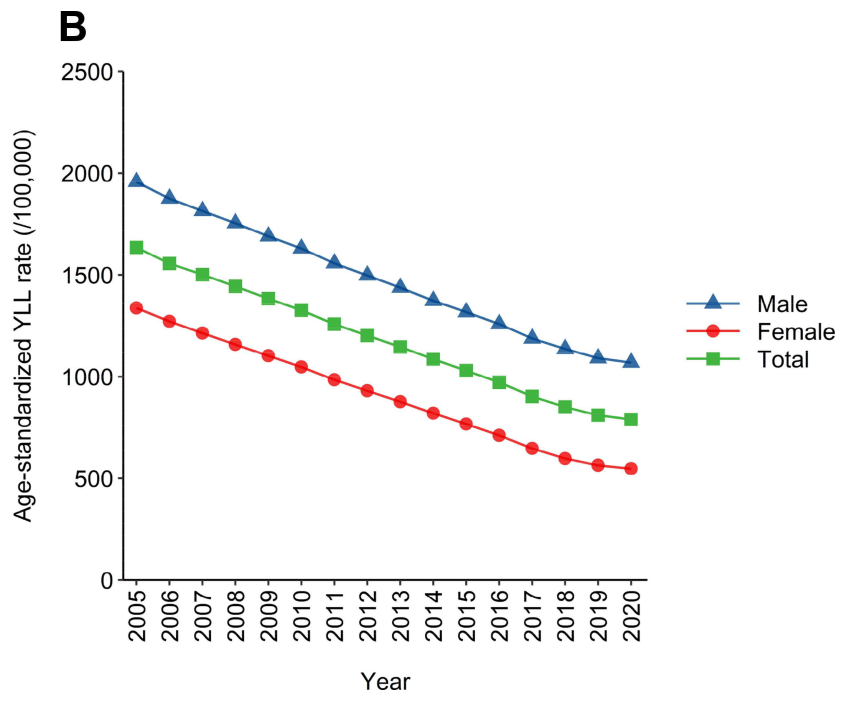

Figure 2 (A) Age-standardized mortality rate for COPD in China, 2005-2020. (B) Age-standardized YLL rate for COPD in China, 2005-2020. Abbreviations: COPD, chronic obstructive pulmonary disease; YLL, years of life lost.

provinces with the greatest contribution to the increase in deaths due to population growth were Tianjin $(52.5 \%)$, followed by Beijing (44.2\%) and Shanghai (39.4\%), and the provinces with the least contribution were Guizhou $(-7.2 \%)$, Sichuan $(-4.0 \%)$ and Heilongjiang $(-1.7 \%)$. The province with the largest contribution to the increase in deaths due to population aging was Heilongjiang (121.3\%), followed by Jilin (103.2\%) and Ningxia $(101.6 \%)$, and the provinces with the least contribution were Tibet (26.1\%), followed by Guangxi $(29.9 \%)$ and Fujian $(38.7 \%)$.

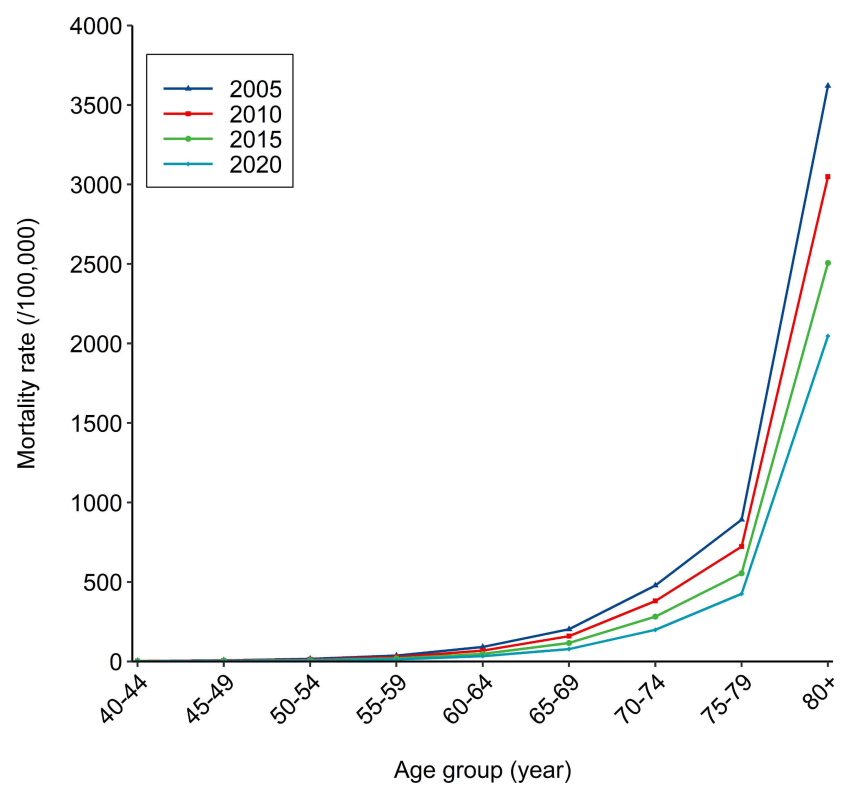

Figure 3 Mortality rate of COPD by age group, 2005, 2010, 2015 and 2020.

\section{Discussion}

We found a remarkable decline of COPD mortality and YLL rate in China during 2005-2020. The decline was seen in almost all provinces. There were substantial variations of COPD mortality rate, ranging from 9.7/100,000 in Jilin to $123.7 / 100,000$ in Sichuan. The magnitude of declining also varied markedly from $23.5 \%$ in Hunan and $77.8 \%$ in Jilin. Only one province (Tibet) showed an increasing trend of COPD mortality during the study period.

The significant declining trend observed in this study may be largely due to the increase of medical and health resources, the development of medical services, the rise of social and economic status, the improvement of people's health literacy and the decrease in exposure to related risk factors such as ambient and household air pollution. ${ }^{14-16}$ China's health care reform has gradually expanded the coverage of health insurance since 2009. The new rural cooperative insurance scheme covered almost all rural areas, which makes it possible for COPD patients, especially in rural areas, to go to hospitals or community health centers for appropriate diagnosis and treatment. ${ }^{17}$ A study showed that compared with 1990, the age-standardized YLL rate of COPD in China in 2019 was 862.4/100,000 with a 74\% decrease, and the YLL of COPD was $15,411,452$ with a $34 \%$ decrease. However, COPD was still the fourth leading cause of YLL in China, accounted for approximately $7 \%$ of the all-cause YLL. ${ }^{1}$ In our study, COPD caused 14,764,545 YLL in China in 2020, consistent with previous literature, ${ }^{1}$ indicating that although the 

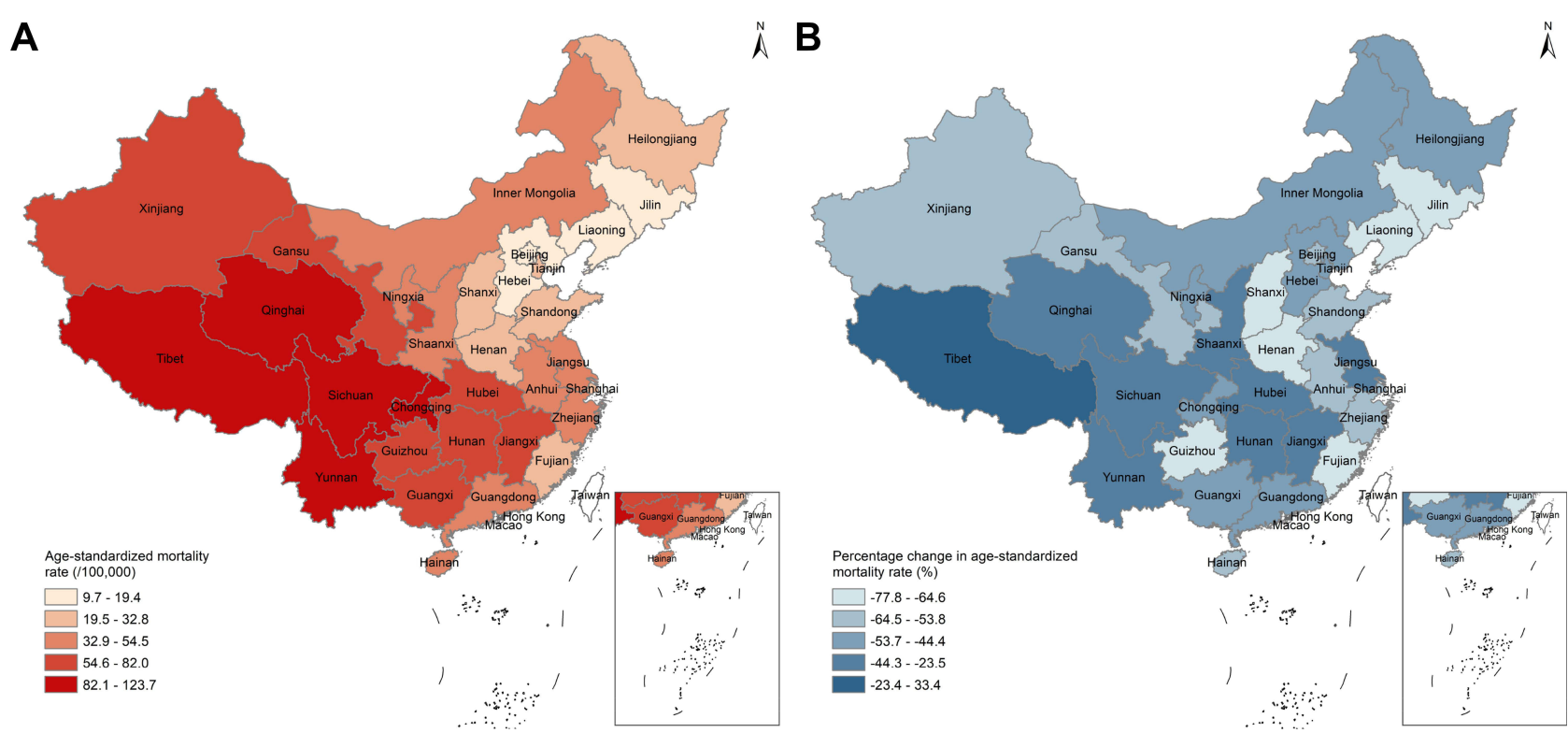

Figure 4 (A) Age-standardized mortality rate of COPD per 100,000 population by province, 2020; (B) the percentage change in age-standardized mortality rate of COPD by province, 2005-2020.

YLL of COPD is a downward trend, it will continue to be a major public health problem.

Reduction of COPD-associated risk factors may also contribute to the declining trend. Previous evidence showed that smoking, ambient and indoor air pollution had an impact on COPD mortality in the Chinese population. ${ }^{18}$ During
2003-2013, the standardized smoking prevalence in China decreased slightly from $26.0 \%$ (95\% CI $25.8-26.2$ ) to $25.2 \%$ (25.1-25.4) in Chinese population aged 15 years or older. ${ }^{19}$ The proportion of COPD deaths attributable to tobacco had a slight decrease from $2005(60.6 \%)$ to $2019(59.3 \%) .{ }^{20} \mathrm{~A}$ study showed that there were considerable decreases in the

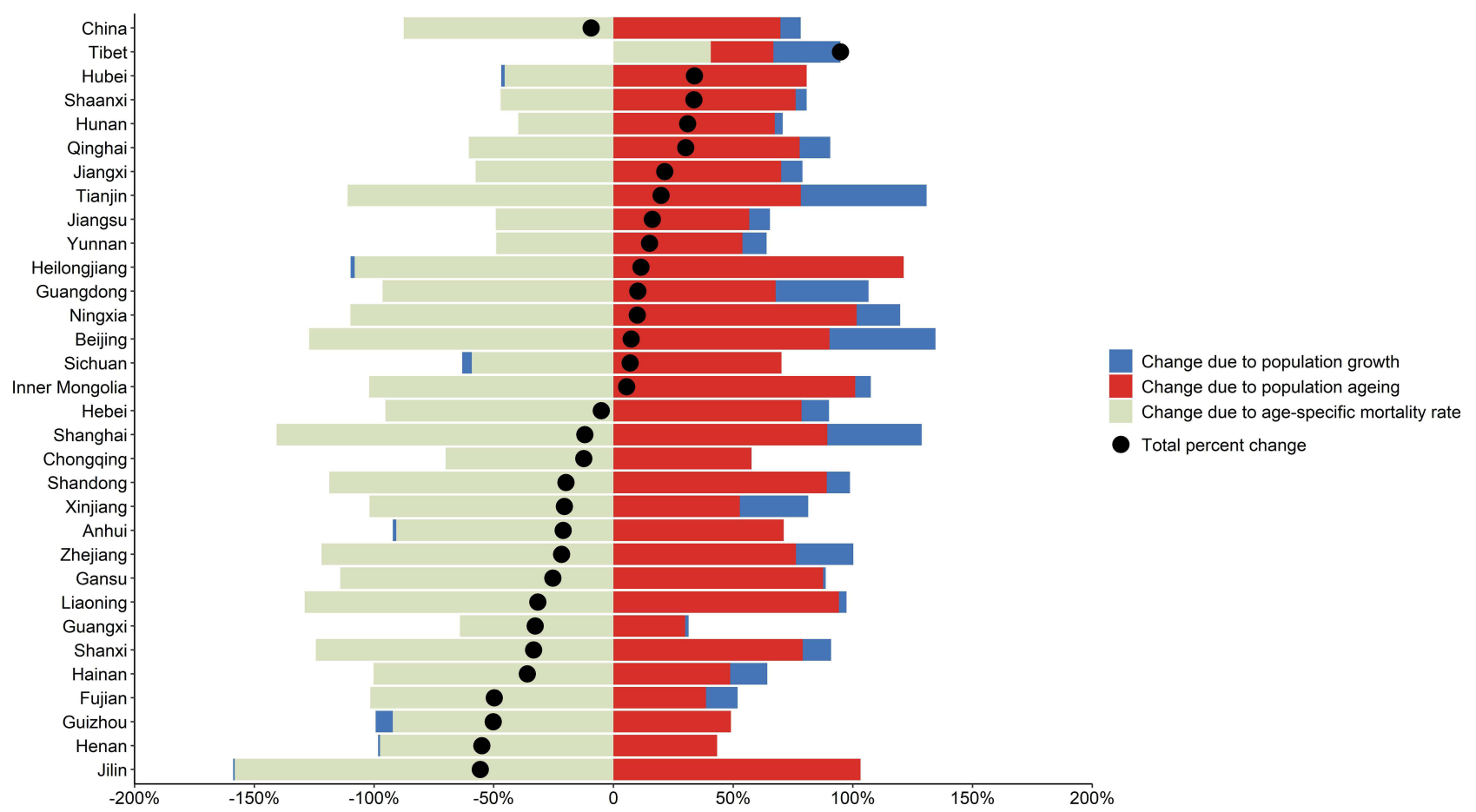

Figure 5 China and 31 provinces decomposition of changes in COPD death due to population growth, population ageing, and changes in age-specific mortality rates, 20052020. 
proportion of COPD deaths attributable to household air pollution from solid fuels from 1990 (40.8\%) to 2019 $(7.6 \%),{ }^{20}$ which is in line with another literature, ${ }^{21}$ and the proportion of COPD deaths attributable to ambient particulate matter pollution had a downward trend after $2015 .^{20}$ Substantial reduction of exposure to occupational risk factors may also be associated with a decrease in the prevalence, morbidity, and mortality of COPD. ${ }^{22}$

The burden of COPD varied considerably among the regions of Asia. The age-standardized mortality rates were 38.8/100,000, 64.6/100,000 and 93.2/100,000 in Southeast Asia, East Asia and South Asia respectively in 2019. ${ }^{1}$ The mortality of COPD in China $(65.2 / 100,000)$ was higher than Japan (7.4/100,000), Mongolia (19.6/100,000) as well as Republic of Korea $(13.2 / 100,000)$ but lower than Democratic People's Republic of Korea $(105.2 / 100,000){ }^{1}$ The substantial variations of health-care policies and the practice of medicine among the regions of Asia have an impact on the burden of COPD. ${ }^{23}$

In consistent with previous studies, we observed a higher mortality rate of COPD in the western provinces compared with the eastern and central provinces. On the one hand, the economic development of the western region is relatively slow, the level of marketization and education is also relatively backward, and the medical facilities and health resources quality are also relatively backward compared with the central and eastern regions. ${ }^{24-26}$ A study also showed that the COPD mortality rates showed a downward trend for all five Sociodemographic index regions between 1990 and 2017, decreasing from regions with a low index to those with a high index. ${ }^{27}$ Moderate to strong negative correlations were seen between the mortality rates and socio-demographic index. ${ }^{28}$ On the other hand, lung cancer, COPD and nasopharyngeal carcinoma were the top three diseases that smoking had the greatest impact on death in both men and women. Compared with other regions, the smoking rate in western region was the highest. ${ }^{29}$ Studies have shown that among the chronic respiratory diseases and cardiovascular and cerebrovascular diseases related to indoor air pollution, COPD is the most influential. The difference of standardized mortality due to indoor air pollution among provinces is related to the use of solid fuels. Residents in western provinces such as Guizhou, Gansu and Qinghai still use solid fuels for heating and cooking, so the attributable mortality is higher. ${ }^{30}$ Besides low detection rate of lung function may cause this result. A large nationwide study in China reported a significantly lower rate of lung function test in areas with household use of polluted fuels than those with clean fuels. ${ }^{31,32}$
In line with the literature, the mortality rate of COPD was higher in males than in females. A study shows that the prevalence of COPD in males in China was much higher than in females. ${ }^{18}$ The excess was most likely to be explained by historic patterns of smoking and occupational exposures in Chinese men. ${ }^{33-35}$ A study found that altitude had a harmful association with COPD, with a dose-response relationship. COPD mortality above $1000 \mathrm{~m}$ was $3-4$ per 10,000 people higher than within $100 \mathrm{~m}$ of sea level. ${ }^{36}$ Tibet is located in the Qinghai Tibet Plateau. Due to the unique climate and geographical environment of the plateau, the air is thin, the oxygen content is low, the temperature difference between day and night is large, and the climate is dry. Therefore, it has a certain impact on respiratory diseases. ${ }^{37}$ Additionally, household air pollution might be an important reason, a study showed that the age-standardized death rate per 100,000 attributable to household air pollution from solid fuels was highest in Tibet (75.8). ${ }^{21}$ Guo and his colleagues showed that prevention of household air pollution and use of alternative fuels are more important strategies for reducing COPD for high altitude regions. ${ }^{38}$ Since the launch of the cause of death surveillance in Tibet, the problem of under-reporting has always existed, ${ }^{39}$ and the problem has been continuously improved by strengthening training at all levels, which may be one of the reasons for the increase in mortality in Tibet. In addition, it may be related to the enhancement of people's health awareness and the increase in lung function detection rate.

Among different provinces, the contribution of the three types of causes to the change of death number is quite different. In developed areas such as Shanghai, Beijing and Tianjin, due to the development of urbanization and population mobility, the contribution of population increase to the increase in death number is at a higher level in the whole country; and the most obvious increase in death number is in the northeast, which indicates that the degree of population aging in this area is relatively high.

Although the mortality rate of COPD decreased substantially, it remains the third leading cause of death in China. ${ }^{1}$ In order to reduce the burden caused by COPD, one specific action for chronic respiratory diseases was included in the Healthy China Action Plan (2019-2030) issued by China State Council. ${ }^{40}$ The objectives of the chronic respiratory disease control and prevention action plan include increase of awareness rate of chronic obstructive pulmonary disease among general population and undertaking lung function test once a year among highrisk population aged 40 years or above. It also advocated 
that the health management of patients with COPD should be included in the national basic public health services.

The present study was also subject to several limitations. Firstly, we counted COPD deaths only when COPD was coded as the underlying cause of death, which might underestimate the true mortality from COPD, because people with COPD often experience multi-morbidity. Secondly, we only indicated the trend of COPD mortality and variations of this trend across different provinces in China without additional data to explain the observed trend and variations. Thirdly, the quality of the coding might be poor in some rural western surveillance sites. Regular training was undertaken at national, provincial and county/district level to the coders and stringent quality control measures are in place in the whole surveillance system. Furthermore, we used garbage code redistribution to ensure the completeness and accuracy of COPD as a cause of death.

\section{Conclusion}

In conclusion, COPD remains an important public health problem in China due to its high mortality and YLL, particularly in western areas and older age groups. The government still needs to actively implement vigorous COPD prevention and control strategies, such as strengthening health education, improving residents' health literacy, strictly implementing tobacco control action, and strengthening early lung function screening of high-risk population, to improve the quality of life of patients and reduce premature deaths due to COPD.

\section{Abbreviations}

COPD, chronic obstructive pulmonary disease; U5MR, under-5 mortality rate; NMSS, National Mortality Surveillance System; URR, under-reporting rate; 45q15, probability of death for adults aged 15-60 years; 5q0, probability of death for children aged under 5 years; MLTFS, Model life table system with flexible standard; ICD, International Classification of Diseases; CI, confidence interval; AAPC, average annual percent change.

\section{Data Sharing Statement}

The data from NMSS and study materials will be made available to other researchers from the corresponding author on reasonable request for purposes of reproducing the results or replicating the procedure.

\section{Ethics Approval}

This study was approved by the ethics committee of the National Center for Chronic and Noncommunicable
Disease Control and Prevention, Chinese Center for Disease Control and Prevention.

\section{Author Contributions}

All authors made substantial contributions to conception and design, acquisition of data, or analysis and interpretation of data; took part in drafting the article or revising it critically for important intellectual content; agreed to submit to the current journal; gave final approval of the version to be published; and agree to be accountable for all aspects of the work.

\section{Funding}

This study was supported by the National Key Research and Development Program of China (2018YFC1315301).

\section{Disclosure}

The authors report no conflicts of interest in this work.

\section{References}

1. Abbafati C, Abbas KM, Abbasi M, et al. Global burden of 369 diseases and injuries in 204 countries and territories, 1990-2019: a systematic analysis for the Global Burden of Disease Study 2019. Lancet. 2020;396 (10258):1204-1222. doi:10.1016/S0140-6736(20)30925-9

2. Yin P, Wang H, Vos T, et al. A subnational analysis of mortality and prevalence of COPD in China From 1990 to 2013 Findings From the Global Burden of Disease Study 2013. Chest. 2016;150(6):12691280. doi:10.1016/j.chest.2016.08.1474

3. Yin P, Feng X, Astell-Burt T, et al. Spatiotemporal variations in chronic obstructive pulmonary disease mortality in China: multilevel evidence from 2006 to 2012. COPD J Chronic Obstructive Pulmonary Dis. 2016;13(3):339-344. doi:10.3109/15412555.2015.1084613

4. Liu S, Wu X, Lopez $\mathrm{AD}$, et al. An integrated national mortality surveillance system for death registration and mortality surveillance, China. Bull World Health Organ. 2016;94(1):46-57. doi:10.2471/ blt. 15.153148

5. Kang G, Peng Y, Lijun W, et al. Propensity score weighting for addressing under-reporting in mortality surveillance: a proof-of-concept study using the nationally representative mortality data in China. Popul Health Metr. 2015:13. doi:10.1186/s12963-015-0051-3

6. Wang Y, Li X, Zhou M, et al. Under-5 mortality in 2851 Chinese counties, 1996-2012: a subnational assessment of achieving MDG 4 goals in China. Lancet. 2016;387(10015):273-283. doi:10.1016/ s0140-6736(15)00554-1

7. Wang H, Dwyer-Lindgren L, Lofgren KT, et al. Age-specific and sexspecific mortality in 187 countries, 1970-2010: a systematic analysis for the Global Burden of Disease Study 2010. Lancet. 2012;380:9859. doi:10.1016/S0140-6736(12)61719-X

8. Naghavi M, Makela S, Foreman K, O’Brien J, Pourmalek F, Lozano R. Algorithms for enhancing public health utility of national causes-of-death data. Popul Health Metr. 2010;1:9. doi:10.1186/1478-7954-8-9

9. Wu S, Gona PN, Martinez G, et al. Global, regional, and national age-sex-specific mortality for 282 causes of death in 195 countries and territories, 1980-2017: a systematic analysis for the Global Burden of Disease Study 2017. Lancet. 2018;392(10159):17361788. doi:10.1016/S0140-6736(18)32203-7

10. Department of Population and Employment \& Statistics National Bureau of Statistics of China. China Population and Employment Statistics Yearbook 2011. Beijing: China Statistics Press; 2012. 
11. Kim HJ, Fay MP, Feuer EJ, Midthune DN. Permutation tests for joinpoint regression with applications to cancer rates. Stat Med. 2000;19(3):335-351. doi:10.1002/(sici)1097-0258(20000215) $19: 3<335:$ :aid-sim336>3.0.co; $2-z$

12. GBD 2015 Mortality and Causes of Death Collaborators. Global, regional, and national life expectancy, all-cause mortality, and cause-specifi c mortality for 249 causes of death, 1980-2015: a systematic analysis for the Global Burden of Disease Study 2015. Lancet. 2016;389(10053):1459-1544. doi:10.1016/S0140-6736(16) 31012-1

13. Das Gupta P. Standardization and Decomposition of Rates: A User's Manual. Washington, DC: US Bureau of the Census; 1993.

14. World Health Organization. Noncommunicable diseases and mental health. NCD global Monitoring Framework [cited May 5, 2021.]; Available from: https://www.who.int/nmh/global_monitoring_frame work/en/. Accessed October 20, 2021.

15. Ruvuna L, Sood A. Epidemiology of chronic obstructive pulmonary disease. Clin Chest Med. 2020;41(3):315-327. doi:10.1016/j. ccm.2020.05.002

16. National Center for Health Statistics. Chronic Obstructive Pulmonary Disease (COPD) includes: chronic bronchitis and emphysema; 2017. [cited 2021 February 21.]; Available from: https://www.cdc.gov/nchs/ fastats/copd.htm. Accessed October 20, 2021.

17. Yip WC, Hsiao WC, Chen W, Hu S, Ma J, Maynard A. Early appraisal of China's huge and complex health-care reforms. Lancet. 2012;1:833-842.

18. Wang C, Xu JY, Yang L, et al. Prevalence and risk factors of chronic obstructive pulmonary disease in China (the China Pulmonary Health CPH study): a national cross-sectional study. Lancet. 2018;391 (10131):1706-1717. doi:10.1016/s0140-6736(18)30841-9

19. Wang M, Luo X, Xu S, et al. Trends in smoking prevalence and implication for chronic diseases in China: serial national cross-sectional surveys from 2003 to 2013. Lancet Respir Med. 2018:35-45. doi:10.1016/S2213-2600(18)30432-6

20. Abbafati C, Abbas K, Abbasi-Kangevari M, et al. Global burden of 87 risk factors in 204 countries and territories, 1990-2019: a systematic analysis for the Global Burden of Disease Study 2019. Lancet. 2020:1223-1249. doi:10.1016/S0140-6736(20)30752-2

21. Yin P, Brauer M, Cohen AJ, et al. The effect of air pollution on deaths, disease burden, and life expectancy across China and its provinces, 1990-2017: an analysis for the Global Burden of Disease Study 2017. Lancet Planetary Health. 2020;4(9):E386E98. doi:10.1016/s2542-5196(20)30161-3

22. Syamlal G, Doney B, Mazurek JM. Chronic obstructive pulmonary disease prevalence among adults who have never smoked, by industry and occupation - United States, 2013-2017. MMWR Morbidity Mortality Weekly Rep. 2019;68(13):303-307. doi:10.15585/mmwr. mm6813a2

23. Tan WC, Ng TP. COPD in Asia: where East meets West. Chest. 2008;133(2):517-527. doi:10.1378/chest.07-1131

24. National Bureau of Statistics of China. China Statistical Yearbook (2016). Beijing: China Statistics Press; 2016.

25. Wang Q, Jin C. Quantitative measurement of economic development imbalance among urban agglomerations in China. J Quantitative Tech Economics. 2018;35(11):77-94. doi:10.13653/j.cnki.jqte.2018.11.005
26. Guo Y, Liu Q. Comprehensive evaluation on regional differences of medical and health development level in China. Chine $J$ Health Statistics. 2016;33(2):251-253.

27. Li X, Cao X, Guo M, Xie M, Liu X. Trends and risk factors of mortality and disability adjusted life years for chronic respiratory diseases from 1990 to 2017: systematic analysis for the Global Burden of Disease Study 2017. Br Med J. 2020;368. doi:10.1136/ bmj.m234

28. Haldun A. User's guide to correlation coefficients. Turkish J Em Med. 2018;18(3):91. doi:10.1016/j.tjem.2018.08.001

29. Liu Y, Liu J, Liu S, et al. Death and impact of life expectancy attributable to smoking in China, 2013. Chine J Epidemiol. 2017;38 (08):1005-1010. doi:10.3760/cma.j.issn.0254-6450.2017.08.002

30. Yin P, Cai Y, Liu J, et al. Disease burden attributable to household air pollution in 1990 and 2013 in China. Chine J Preventive Med. 2017;51(01):53-57. doi:10.3760/cma.j.issn.0253-9624.2017.01.011

31. Fang L, Bao H, Wang B, et al. Survey and analyses of rate of spirometry examination in adults aged 40 years and older in China,2014. Chine J Epidemiol. 2018;39(05):593-599. doi:10.3760/ cma.j.issn.0254-6450.2018.05.010

32. Lv X, Cong S, Fan J, et al. Analyses of the rate of spirometry examination and its related factors in chronic obstructive pulmonary disease patients aged 40 years or older in China, 2014-2015. Chine J Epidemiol. 2020;(05):672-677. doi:10.3760/cma.j.cn11233820200122-00054

33. Adeloye D, Chua S, Lee C, et al. Global and regional estimates of COPD prevalence: systematic review and meta-analysis. J Glob Health. 2015;5(2):020415. doi:10.7189/jogh.05-020415

34. Fang X, Wang X, Bai C. COPD in China: the burden and importance of proper management. Chest. 2011;139(4):920-929. doi:10.1378/ chest.10-1393

35. Silverman EK, Weiss ST, Drazen JM. Gender-related differences in severe, early-onset chronic obstructive pulmonary disease. Am J Respir Crit Care Med. 2000;162(6):2152. doi:10.1164/ ajrccm.162.6.2003112

36. Ezzati M, Horwitz MEM, Thomas DSK, et al. Altitude, life expectancy and mortality from ischaemic heart disease, stroke, COPD and cancers: national population-based analysis of US counties. $J$ Epidemiol Community Health. 2012;66(7):e17. doi:10.1136/ jech.2010.112938

37. Guan W, Ge R. Effects of plateau environment on respiratory diseases. Natl Med China. 2015;95(30):2491-2493. doi:10.3760/cma.j. issn.0376-2491.2015.30.022

38. Guo Y, Xing Z, Shan G, et al. Prevalence and risk factors for COPD at high altitude: a large cross-sectional survey of subjects living between 2100-4700 m above sea level. Front Med. 2020:7. doi:10.3389/fmed.2020.581763

39. Bai G, He F, Hu Y. Death causes of residents in Tibet autonomous region. Disease Surveillance. 2011;26(01):55-57. doi:10.3784/j. issn.1003-9961.2011.01.017

40. General Office of the State Council. Healthy China Action (20192030); 2019. [cited May 5, 2021.]; Available from: http://www.gov. cn/xinwen/2019-07/15/content 5409694.htm. Accessed October 20, 2021.

\section{Publish your work in this journal}

The International Journal of COPD is an international, peer-reviewed journal of therapeutics and pharmacology focusing on concise rapid reporting of clinical studies and reviews in COPD. Special focus is given to the pathophysiological processes underlying the disease, intervention programs, patient focused education, and self management protocols. This journal is indexed on PubMed Central, MedLine and CAS. The manuscript management system is completely online and includes a very quick and fair peer-review system, which is all easy to use. Visit http://www.dovepress.com/testimonials.php to read real quotes from published authors. 